\title{
Semantische Analyse des Kausalkon- nektors weil in historischen Texten im Fremdsprachenunterricht
}

\author{
Erzsébet Szabó - Ervín Weiss - Eva Stranovská
}

\begin{abstract}
Semantic analysis of causal conjunction weil in historical texts in foreign language teaching

Abstract

This article deals with pragmalinguistic typology which helps to represent the cognitive level. Thanks to the pragmalinguistic typology, one can analyze the communication process in a more detailed way and detect that translating and stringing together translated sentences is not enough for the respondent to understand what the speaker wants to express. For this purpose, it is very important to know how to produce syntactically and semantically meaningful sentences as well as how to use adequate linguistic and non-linguistic means. The main aim of this article is to point out the importance of pragmalinguistic typology, which plays an essential role to describe semantically the causal constructions in historical texts.
\end{abstract}

Keywords: pragmalinguistic typology; historical texts; semantic analysis; causality Schlüsselwörter: pragmalinguistische Typologie; historische Texte; semantische Analyse; Kausalität

Subject-Affiliation in New CEEOL: Social Sciences - Education - School Education

DOI: 10.36007/eruedu.2021.3.035-043

\section{Einleitung}

Der vorliegende Artikel beschäftigt sich vor allem mit der semantischen Analyse von historischen Texten aus dem ältesten Stadtbuch von Schmöllnitz 1410-1735. Der Beitrag untersucht den Kausalkonnektor weil auf der Grundlage der pragmalinguistischen Typologie von Eve Sweetser, die in erster Linie den Fremdsprachenunterricht im tertiären Bildungsbereich betrifft. Auch historische Texte können beim Erlernen einer Fremdsprache als wertvolle Hilfsmittel dienen und es ist wichtig, sie dabei so zu gestalten, dass sie das Interesse der Studierenden wecken. Mit Hilfe einer semantischen Analyse der in einem Text verwendeten Konnektoren kann der Schwierigkeitsgrad dieses Textes eingeschätzt werden und sie kann auch zur Entwicklung der Lesefertigkeit bei den Studenten beitragen. 


\section{Beschreibung der kognitiven Theorie von Eve Sweetser}

Viele syntaktisch ausgerichtete Forschungsvorhaben werden heute auf der Grundlage von semantischen Regeln und Prinzipien durchgeführt, die sich auf die kognitive Theorie von Eve Sweetser beziehen.

Sweetsers Lehre geht von den Thesen des Linguisten George Lakoff (1980, 52-53) und des Philosophen Mark Johnson (1980, 52-53) aus, aufgrund deren sich viele Fälle von Polysemie auf die metaphorische Funktionsweise des kognitiven Apparats zurückführen lassen. Der Grundgedanke des kognitiv orientierten Ansatzes, der für Sweetsers Theorie charakteristisch ist, kann folgendermaßen beschrieben werden: Die einzelnen lexikalischen Einheiten haben nicht nur eine konkrete Bedeutung, sondern mit Hilfe von kognitiv-metaphorischen Prozessen können auch ihre abstrakten Verwendungsweisen erklärt werden. Nach Ansicht von Lakoff und Johnson können das Verstehen, Verwenden und Produzieren von sprachlichen Strukturen als wichtiger Bestandteil der Sprachkompetenz betrachtet werden (Ferraresi 2011, 129-130; Lakoff, Johnson 1980, 52-53).

Laut Sweetsers kognitiver Theorie $(1990,52)$ lässt sich eine Äußerung auf drei kognitiven Verknüpfungsebenen interpretieren:

- auf der propositionalen Ebene (Sachverhaltsebene) - diese Ebene wird als Basisebene betrachtet, auf der die Beziehung zwischen zwei Propositionen im Sinne eines Grund-Folge-Verhältnisses hergestellt wird.

- auf der epistemischen Ebene - auf dieser Ebene gibt es einen klaren Bezug zum Sprecher selbst, der den Inhalt des Satzes im übergeordneten (externen) Konnekt als seine Behauptung kenntlich macht oder allgemeingeltende Werturteile ausdrückt.

- auf der sprechaktbezogenen Ebene - auf dieser Ebene bezieht der Sprecher die Stellungnahme auf sich selbst wie auch auf die epistemische Ebene. Der Unterschied zur epistemischen Ebene besteht darin, dass auf der sprechaktbezogenen Ebene ein Sprechakt abläuft, wobei das untergeordnete (interne) Konnekt den Grund für das übergeordnete (externe) Konnekt ausdrückt (Breindl, Volodina, Waßner 2014, 1125; Gagel 2015, 56; Sweetser 1990, 52).

Für die oben beschriebenen Verknüpfungsebenen sind unterschiedliche Indikatoren charakteristisch, die als wichtige Hilfsmittel dazu dienen, den Text besser und tiefer zu verstehen. Solche Indikatoren drücken vor allem das Vorhaben des Sprechers aus und umfassen u. a. Modalverben, Modalpartikel, Adverbien und Verben.

Anhand der pragmalinguistischen Typologie kann man den Kommunikationsprozess näher untersuchen und ist nicht mehr ausschließlich auf die Untersuchung der linguistischen Kompetenz beschränkt. Für die Vermittlung von Fremdsprachen rückt die Bedeutung der Ausbildung von kommunikativer Kompetenz in den Vordergrund. Ein kompetenter Sprecher muss mit Blick auf die einzelnen Ebenen wissen, wie man gut strukturierte Sätze mit angemessener Wortwahl produziert, die syntaktisch und semantisch akzeptabel sind. Außerdem muss er/sie darauf achten, wie er/sie sich verhält und welche sprachlichen und außersprachlichen Mittel er/sie verwendet, damit der Rezipient genau das versteht, was er/sie mit- 
teilen möchte. Zu diesem Zweck wird im vorliegenden Artikel analysiert, welche Relation es, zwischen den Zeichen und denen, die sie benutzen, gibt (Hufeisen, Neuner 1999, 35-43).

\section{Kausalität im Deutschen}

Das Wesen der Kausalrelation besteht darin, dass mit Hilfe kausaler Konnektoren zwei gekennzeichnete Sachverhalte, die den Grund und die daraus resultierende Folge bezeichnen, miteinander verknüptt werden. (Weiss 2016, 17)

Für Sprachhistoriker ist es wichtig, die semantischen Funktionen von Antezedens und Konsequens zu erfassen, mit deren Hilfe man zwischen antezedensmarkierenden und konsequensmarkierenden Kausalkonnektoren unterscheiden kann. Antezedensmarkierende Kausalkonnektoren drücken aus, dass der Sachverhalt im internen (subordinierten) Konnekt eine Ursache des Sachverhalts darstellt, der im externen Konnekt zum Ausdruck gebracht wird. Konsequensmarkierende Kausalkonnektoren drücken dagegen eine Folge des Sachverhalts im externen (übergeordneten) Konnekt aus (Brendl, Volodina, Waßner 2014, 792).

Das oben beschriebene Antezedens-Konsequensverhältnis kann man auf temporale Relationen zurückführen, die als Basis für die Entwicklung weiterer kausaler Bindewörter dienen. Nach Dal und Eroms $(2014,256)$ stellt die begründende Bedeutung eine so genannte „Folgerungsbeziehung” dar, die bei der temporalen Verbindung von argumentativen Sachverhalten angeführt wird. Darüber hinaus kann man feststellen, dass die kausale Bedeutung durch unterschiedliche Metonymisierungs- und Metaphorisierungsprozesse zahlreicher verschiedene Konjunktionen herausgebildet hat (Dal, Eroms 2014, 256).

\section{Semantische Analyse kausaler Konstruktionen in dem ält- esten Stadtbuch von Schmöllnitz (1410-1735) im Kontext der Pragmalinguistik}

\subsection{Untersuchungsumfeld und Untersuchungsziel}

Im praktischen Teil des Artikels geht es um die semantische Analyse des Kausalkonnektors weil, die anhand von historischen Texten aus dem ältesten Stadtbuch von Schmöllnitz (1410-1735) durchgeführt wird. Dabei wird die in Abschnitt 2 vorgestellte pragmalinguistische Typologie verwendet, die drei Verknüpfungsebenen (propositionale, epistemische, sprechaktbezogene Ebene) umfasst, denen die in den Texten vorkommenden Sätze mit dem kausalen Bindewort weil zugeordnet werden. Das Untersuchungsziel ist, die Kausalkonstruktionen mit Hilfe der Verknüpfungsebenen semantisch zu analysieren.

\subsection{Untersuchungsmaterial}

Als Untersuchungsmaterial werden Texte (Rechtstexte, Verträge, Testamente, Entscheidungen und Rechtsprechungsakte des Stadtrates, Statuten und unterschied- 
liche Ordnungen) aus dem ältesten Stadtbuch von Schmöllnitz (1410-1735) herangezogen, in denen die Kausalkonjunktion weil in verschiedenen Formen auftritt. Das zugrunde gelegte Material ist erst vor kurzem editiert worden und wurde bisher noch nicht von anderen Linguisten semantisch analysiert. Die transkribierte Edition des Stadtbuchs wurde von Miroslav Lacko und Erika Mayerová (2016) vorgenommen. Die Originalhandschrift des Stadtbuches befindet sich heute in der Handschriftensammlung der Széchenyi Bibliothek in Budapest. Das untersuchte Protokollbuch ist eine so genannte Papierhandschrift mit 194 Blättern, die v. a. deutsche und lateinische Eintragungen enthält, daneben kommt auch ein Teil der Schrift in slowakisierter tschechischer Sprache vor (Lacko, Mayerová 2016, 9, 50).

\subsection{Untersuchungsmethode}

Während der Untersuchung wird eine qualitative Inhaltsanalyse (vgl. Bortz, Döring 2006, 332) der Texte vorgenommen, in deren Verlauf die Sinneinheiten, die den Kausalkonnektor weil enthalten, kategorisiert werden. Aufgrund der Inhaltsanalyse lässt sich feststellen, auf welcher Verknüpfungsebene die verschiedenen Varianten der Kausalkonjunktion weil in den Texten anzusiedeln sind.

\subsection{Inhaltsanalyse}

In diesem Teil des Beitrags werden die einzelnen Verknüpfungsebenen charakterisiert und mit entsprechenden Beispielsätzen aus dem untersuchten Stadtbuch belegt.

Von einer Verknüpfung auf propositionaler Ebene spricht man dann, wenn das externe Konnekt ein Reportivum enthält. Das heißt, dass die Aussage einen konstativen Charakter hat. Laut Gagel $(2015,29)$ gehören zu Reportiva Berichte, Bestätigungen, Beschreibungen, Feststellungen, Schilderungen oder Bekanntmachungen. Indikatoren für Reportiva sind z. B. Verben im Indikativ, vergangenheitsbezogene Tempusformen und adverbiale Ergänzungen der Zeit (Gagel 2015, 29; Weiss 2016, 22).

Dÿ weÿl aber der erbar herr Caspar Saẅer der nagst nachpar dar czu gewesen ist, so hatt er den obbemelten florian nach erkentniß des rechtens von benimpten kauff abgedrungen... (S. 147, fol. 79r)

...der hans Antel aber hatt fur vns mit warhafftigen Zeugenn genugsam bezeygett, das sie eine solche ist, wie ihr denn vonn ihm vorgeworffen ist wordenn, Derhalbenn sie sich weil sie schuldig ihnn der sachenn war, gegen ihn demutigett vnndt ihnn durch erliche leut vorbitt ersuchett... (S. 158, fol. 90r)

Der letzte Beispielsatz enthält auch ein Pronominaladverb (derhalbenn), das als Indiz für Korrelatkonstruktionen auf der propositionalen Ebene gilt. Nach Gagel $(2015,174)$ wurden Korrelatkonstruktionen mit Pronominaladverbien wie derowegen, derhalben oder derhalben so als Indikatoren für das Vorliegen einer propo- 
sitionalen Lesart gewertet (Gagel 2015, 174).

Epistemische Lesart liegt vor, wenn das externe Konnekt entweder ein Estimativum, ein Evaluativum oder ein Identifikativum beinhaltet. Laut Gagel (2015, 29) sind Estimativa typischerweise solche Aussagen, die eine Einschätzung, eine Behauptung, eine Vermutung oder eine Annahme zum Ausdruck bringen. Charakteristische Merkmale der Estimativa sind v. a. epistemisch verwendete Modalverben (Gagel 2015, 29).

Weil bißher einer den andern harth an Lehn eingeseßen ist, Sol der Bergmeister dieses in Keinen weg zugeben... (S. 168, fol. 104v)

...weil er aber solche nicht hatt abzulegen gehabt, hatt er müssen bey der Gnädigen Obrigkeit Suppliciren Vmb solche zu erlegen... (S. 240, fol. 165r)

Der folgende Subtyp ist das Evaluativum, dessen Vertreter solche Aussagen sind, die Bewertungen oder Werturteile bezeichnen. Typische Indikatoren für Evaluativa sind evaluative Adjektive, Substantive und Verben (Gagel 2015, 29).

...über das weil dieses orts nicht viel Roß vnd furleute sein, hat gedachter Herr Anthonius beÿ vnserm Gnädigen vnd wohlgebornen Herrn, Herrn Christoph Thurzonen angelangt, daß ihro Gnaden an ihre vnterthanen im grundt schreiben vnd denen befehlen wollen... (S. 183, fol. 117r)

...der Ehrwürdige Achtbare vnd wollgelarte Herr Heinricus Leonhardj damalß trewfleißiger Seelsorger der Christlichen Gemeine inn Schmölnitz es vor gutsam vnd rathsam angesehen, weil ihn der Höchste durch seinen gnaden Segen Erben beschehret... (S. 216, fol. 147r)

Der letzte Subtyp ist das Identifikativum, das Hoffnungen oder Gefühle des Sprechers zum Ausdruck bringt. Zu den Indikatoren für Identifikativa gehören Adverbien wie glücklicherweise, hoffentlich oder leider (Gagel 2015, 29). Im hier untersuchten Material fanden sich keine Beispiele für diesen Illokutionstyp.

Die Präsenz verschiedenartiger Modalitätsmarker hilft auch die pragmatische Funktionalität zu beschreiben. Als Modalitätsmarker kann man die Modalverben anführen, die in erster Linie auf epistemische Lesart hindeuten. Sie werden vom Sprecher zum Ausdruck von objektiver oder subjektiver Modalität verwendet (Weiss 2016, 21).

...Daneben hatt er auch hintersich gelassen zimliche schulden be der Handlung Nemlich fl. 221 weil aber diese schulden nirgend anders herhaben können gezahlt werden alls Von dem Haus... (S. 194, fol. 128r)

Zu den Modalitätsmarkern zählt man auch die Modalpartikeln denn, doch, ja, 
nur, wohl und solche Modalitätsformen wie die Konstruktionen mit sein/haben + zu + Infinitiv. Die erwähnten Modalitätsformen sind auch für die epistemische Lesart charakteristisch (Gagel 2015, 133-135; Weiss 2016, 21).

Lobet dem Herren inn seinen Heyligthumb, lobet Ihnn in der Veste seiner macht, lobet Ihn in seinem Thatten, lobet Ihn in seiner großen herrligkeitt. Weil dann auch Vnsere Liebe Kürche Vnndt Gottes Hauß, nicht allein außwendig, sondern auch inwendig, Zimlichen, in abnehmen kommen... (S. 164, fol. 101v)

Die dritte Verknüpfungsebene stellt die illokutive (sprechaktbezogene) Ebene dar, die oft durch die sog. performativen Verben erkennbar ist. Zu den performativen Verben gehören anlegen, auflegen, bezeugen, verobligieren oder vornehmen. Auf der sprechaktbezogenen Ebene sind daneben auch die folgenden Illokutionstypen anzusiedeln: Direktiva, Optativa, Interrogativa, Kommissiva, Expressiva und Deklarativa (Weiss 2016, 22).

Als Direktiva werden nach Gagel $(2015,26)$ in erster Linie Befehle und Aufforderungen bezeichnet. Der Hörer soll sich vom Sprecher zur Ausführung einer zukünftigen Handlung verpflichtet fühlen. Das Direktivum kann durch Verben wie anordnen, auffordern, befehlen, bitten, fordern, fragen oder raten ausgedrückt werden (Gagel 2015, 26).

Mit meinem herrn Schweher, Erhard Sawr Meiner hawsfrawen Stieffvatter, der den mein hausfrawen Salomea Erberlichn Erczogn hath von khindt Auff, Biss in dass Sechtczehndt Jar, Alss Seine leybliche tochter, dass dank ich im mitt sambt meiner hausfrawen Als vnserm rechtn vatter, vnd wellen got vor in b ttn weyll wir leben... (S. 129, fol. 52v)

...so ist doch her Triebell mit der entshuldigung nicht czu frieden gewest sondern mit vleis bies auff ernemte zeit Im Christmonat der sachen nach gefraget weil den durchaus nirgentt kein beweis oder anczeigung ist... (S. 162, fol. 98v)

Laut Gagel $(2015,26)$ werden mit Optativa Wünsche zum Ausdruck gebracht, wie dies das folgende Beispiel zeigt:

Mehr hat er von ihm begeret zuo Empfangen dreÿ felder neben der vorgemelten gang ins Hangendt Hinnauff, weil auch diese felder in freÿem gewesen... (S. 187, fol. 120v)

Interrogativa sind Fragesätze, und zwar entweder Entscheidungs- oder Ergänzungsfragen.

Der Illokutionstyp „Kommissivum“ umfasst v. a. solche Sätze, in denen der Sprecher sich selbst verpflichtet, eine zukünftige Handlung auszuführen. Verben, die mit diesem Illokutionstyp zusammenhängen, sind: anbieten, drohen, schwören, vereinbaren, sich verpflichten, versprechen und wetten. 
Als Expressiva werden solche Sätze bezeichnet, in denen der Sprecher seine eigene Ansicht zu einem Sachverhalt ausdrückt. Expressiva können mit Verben wie danken, sich entschuldigen, gratulieren, klagen oder wünschen realisiert werden.

Deklarativa stellen Sätze dar, durch die der Sprecher einen bestimmten Zustand in der Wirklichkeit herstellt. Dieser Illokutionstyp kann durch Verben wie abdanken, entlassen, ernennen, kundigen, trauen oder verurteilen zum Ausdruck gebracht werden (Gagel 2015, 26-27). Belege mit den oben angeführten Illokutionstypen waren in dem Stadtbuch von Schmöllnitz nicht zu finden.

Auch Korrelate werden als wichtiges Hilfsmittel betrachtet, um die semantischen Merkmale von Konjunktionen zu bestimmen. Neben den oben angegebenen Illokutionstypen ist die sprechaktbezogene Lesart präsent, wenn in den Sätzen Korrelate auftreten (Weiss 2016, 19).

Weil sich nun also die Sache in der Warheit befindet, so hat es ein ordentliches BergRecht, (allwo auch auß der löblichen gemeine der Älteste darbey gewesen) gefunden Vnd beshloßen daß laut der Abziehung Vnd obshienen der löblichen Handlung die lehen, samb den ertzen zufälle... (S. 232, fol. 159v) (Lacko und Mayerová 2016)

\section{Zusammenfassung}

In der vorliegenden Untersuchung wurde die kognitive Ebene von ausgewählten Belegstellen aus dem ältesten Stadtbuch von Schmöllnitz (1410-1735) mit Hilfe einer pragmalinguistischen Typologie beschrieben. Besondere Aufmerksamkeit wurde dabei dem Kausalkonnektor weil gewidmet, weil dieses Bindewort in dem untersuchten Material am häufigsten vorkommt. Die Sätze mit dem Kausalkonnektor weil wurden aus den untersuchten Texten ausgeschrieben und auf der Grundlage der pragmatischen Typologie analysiert. Das heißt, dass die Kausalsätze unter Zuhilfenahme der Indikatoren zu den jeweiligen Verknüpfungsebenen zugeordnet und ihre Bedeutungen beschrieben wurden.

Aufgrund des analysierten Materials ließ sich feststellen, dass die einzelnen Verknüpfungsebenen einen unterschiedlichen Schwierigkeitsgrad haben. Die propositionale Ebene ist vor allem neutral. Das bedeutet, dass in diesen Sätzen keine besonderen Indikatoren vorkommen. Dagegen verlangt die Wahrnehmung der epistemischen und der sprechaktbezogenen Lesart ausreichende, sowohl grammatische als auch syntaktische Vorkenntnisse seitens des Sprachwissenschaftlers. Man sollte die Modalverben, die Modalpartikeln und auch die Adverbien kennen und wissen, wie sie im Rahmen der epistemischen Verknüpfungsebene auftreten. Außerdem ist es genauso wichtig, die Bedeutung der einzelnen Verben im Text zu identifizieren und zu verstehen, was ausschlaggebend beim Erfassen der sprechaktbezogenen Ebene ist.

Es gibt nicht so viele Untersuchungen, die sich mit den historischen Texten im 
Hinblick auf die pragmatische Typologie beschäftigen. Den Kausalkonnektor weil hat vor allem Sebastian Gagel (2015) in seiner Dissertationsschrift „Frühneuhochdeutsche Konnektoren“ auf die Modalität hin untersucht und seine semantische Beschreibung auf der Grundlage der pragmalinguistischen Typologie dargestellt.

Die Analyse historischer Texte zeigt ein hohes Niveau der Sprach- und Lesekompetenz bei Lernenden. Sie sollen kognitive Prozesse anwenden, wie Informationsverarbeitung durch Deduktion und Interpretation des Gelesenen (Liptáková, Cibáková 2013, 13), um den Sinn des Textes verstehen zu können, weil in den frühneuhochdeutschen Texten keine Interpunktionszeichen gebraucht wurden. Daneben wird es vermutet, dass sich durch historische Textanalyse so genannte Sprachvergleichskompetenz bei Lernenden herausbildet (Reimann 2014, 11). Die historischen Texte sollen einen positiven Einfluss auf die Studenten haben und innen bei anspruchsvollen Textarbeiten in der Tertiärbildung Hilfe leisten.

\section{Acknowledgement}

Diese Arbeit wurde von der Agentur für Forschung und dem Vertrag APVV-17-0071 und VEGA 1/0062/19 unterstützt.

\section{Literaturverzeichnis}

Bortz, Jürgen/Döring, Nicola (2006): Forschungsmethoden und Evaluation für Humanund Sozialwissenschaftler. Heidelberg: Springer Medizin Verlag.

Breindl, Eva/Volodina, Anna/Waßner, Hermann Ulrich (2014): Handbuch deutscher Konnektoren 2: Semantik der deutschen Satzknüpfer. Berlin - New York: de Gruyter.

Dal, Ingrid/Eroms, Hans-Werner (2014): Kurze deutsche Syntax auf historischer Grundlage. Berlin - New York: de Gruyter.

Ferraresi, Gisella Ed. (2011): Konnektoren im Deutschen und im Sprachvergleich: Beschreibung und grammatische Analyse. Tübingen: Gunter Narr Verlag.

Gagel, Sebastian (2015): Frühneuhochdeutsche Konnektoren - Entwicklungslinien kausaler Verknüpfungen auf dem Gebiet der Modalität. Diss. masch. Nürnberg: Universität Erlangen.

Hufeisen, Britta/Neuner, Gerhard (1999): Angewandte Linguistik für den fremdsprachlichen Deutschunterricht. Fernstudieneinheit 16. Kassel - München - Tübingen: Universität Kassel.

Lacko, Miroslav/Mayerová, Erika (2016): Das älteste Stadtbuch von Schmöllnitz 14101735. Eine Quelle zu den mitteleuropäischen wirtschaftlichen Verflechtungen. Limbach: Verlag Slovenská spoločnost' pre sociálne a hospodárske dejiny (Slowakische Gesellschaft für Sozial- und Wirtschaftsgeschichte).

Lakoff, George/Johnson, Mark (1980): Metaphors we live by. Chicago: University of Chicago Press.

Liptáková, Ĺudmila/Cibáková, Dana (2013): Edukačný model rozvíjania porozumenia textu v primárnom vzdelávaní. Prešov: Prešovská univerzita v Prešove, Pedagogická fakulta. 
Reimann, Daniel (2014): Kontrastive Linguistik und Fremdsprachendidaktik Iberoromanisch - Deutsch. Romanistische Fremdsprachenforschung und Unterrichtsentwicklung. Tübingen: Narr Verlag.

Sweetser, Eve (1990): From etymology to pragmatics. Metaphorical and cultural aspects of semantic structure. Cambridge: Cambridge University Press.

Weiss, Ervín (2016): Das Stadtbuch von Schwedler - Möglichkeiten der Konnektorenforschung. Nitra: Filozofická fakulta. Univerzita Konštantína Filozofa v Nitre. 